\title{
Cambios en los usos de suelo en la comarca del Bierzo (1990-2012)
}

Recibido: 3 outubro 2017 / Aceptado: 4 setembro 2018

(C) Universidade de Santiago de Compostela 2018

\begin{abstract}
Resumen El paisaje de la comarca del Bierzo, en el noroeste de España, ha experimentado una serie de cambios durante las últimas décadas. Este trabajo tiene como objetivo principal proporcionar un análisis exhaustivo de los cambios de uso/cobertura del suelo en esta comarca utilizando Corine Land Cover durante dos períodos diferentes (1990-2000 y 2000-2012). Esto nos permite analizar las principales tendencias y procesos a los que se vio sometido este territorio. Los principales hallazgos tienen relación con los procesos de abandono que sufre esta comarca, motivado por los cambios en hectáreas totales hacia matorral, procedentes sobre todo de los usos forestales y agrícolas. Casi la mitad de la superficie pertenece al dominio del matorral en 2012, al ocupar en torno a 151000 hectáreas. En otro orden se sitúa el aumento de las plantaciones de chopos por reforestación que también ha sido importante sobre el conjunto, provocando la expansión de las frondosas en la zona. Otros procesos como la intensificación productiva se han producido cerca de las infraestructuras de regadío, aunque no son tan importantes sobre el total regional.
\end{abstract}

Palabras clave Corine - Abandono - Cambios de uso · Intensificación-extensificación · Tablas de contingencia.

David Fernández Nogueira · Eduardo José Corbelle Rico Laboratorio do Territorio. Departamento de Enxeñería Agroforestal.

Universidade de Santiago de Compostela. Escola Politécnica Superior de Enxeñería, Benigno Ledo s/n, 27002 - Lugo (España).

Email: david.fernandez.nogueira@usc.es

Telefono: +34982 823324

\section{Land use change in Bierzo region (1990-2012)}

Abstract The landscape of the Bierzo region, located in northwestern Spain, has experienced a series of transformations along the last decades. The main objective of this work is to provide a comprehensive analysis of land changes using Corine Land Cover for two different time periods (1990-2000 and 2000-2012). This allowed us to analyze the main trends and processes to which this territory was subjected. The main findings are related to the abandonment processes suffered in this region, motivated by changes in total hectares towards shrubland, mainly from the forestry and agricultural uses. Almost half of the total surface belongs to shrubland domain in 2012, occupying around 151000 hectares. On the other hand, the increase of poplars by reforestation processes has also been important, causing the expansion of the hardwoods in the area. Other processes such as productive intensification, appears near the irrigated structures, although they are not so important on the regional total.

Key words Corine - Abandonment - Land use changes · Intensification-extensification · contigency tables.

\section{Introducción}

La monitorización y observación de los cambios producidos en los usos/cobertura de suelo siguen teniendo a día de hoy gran repercusión dentro del ámbito científico multidisciplinar (Levers et al., 2016; Meyfroidt et al., 2013; Turner et al., 2007). Esto nos sirve para conocer e interpretar con detalle la evolución y las fuerzas motrices de cualquier territorio, donde principalmente son las alteraciones que se producen en los usos urbanos, forestales, agrícolas y zonas naturales los de mayor importancia. En ocasiones las variaciones en las cubiertas del suelo aparecen como respuesta frente a la falta de estabilidad de distintos factores: socioeconómicos, políticos, tecnológicos e incluso culturales a los que se ven sometidos diferentes territorios (Corbelle et al., 2015; de Aranzabal et al., 2008; Plieninger et al., 2016; van Vliet et al., 2015). 
El conocimiento sobre el grado de ocupación y las tipologías de uso es esencial también para interpretar la sostenibilidad y el desarrollo de cualquier región (OSE, 2006). La preocupación de diferentes organismos internacionales como la Food and Agriculture Organization o la European Environment Agency (2007) se centra y es latente en el estudio de los "land use systems" que analizan los recursos naturales disponibles, la ecología del paisaje, el cambio climático o mismamente los servicios ecosistémicos asociados a los distintos usos, que cada vez se encuentran más globalizados (Lambin et al., 2003).

En las últimas décadas, las cubiertas en España han estado muy condicionadas por las políticas europeas de la PAC (Jones et al., 2011; Corbelle et al., 2015; MacDonald et al., 2000; Molinero et al., 2013), modificando el paisaje agrario y forestal principalmente. Las subvenciones y regulaciones hacia ciertos cultivos o la reforestación de distintas especies forestales tienen un gran impacto como fuerzas de cambio. También, la aparición de las Denominación de Origen Protegida (DOP) han tenido gran importancia en el mantenimiento de algunas cubiertas, condicionando la evolución de la estructura socio-económica y fomentando el cooperativismo entre los propietarios. Gracias a las DOP se produce la modernización de determinados sectores productivos, donde se mejora el comercio y la exportación de productos ligados a la horto-fruticultura (viñedo, cerezo, manzanas reineta, pimiento rojo).

Algunas de las principales tendencias observadas en los trabajos que analizan cambios de uso/cobertura en el Norte y Noroeste de la Península Ibérica: tratan la intensificación y extensificación productiva en los usos agrícolas (Alberdi, 2001; Lasanta, 2000; Lomba et al., 2011; Serrano et al., 2002), el abandono de usos (Corbelle \& Crecente, 2008), la artificialización y crecimiento urbano (Jaraíz et al., 2012; Serra et al., 2014) y también los cambios en la composición de las masas forestales (Martínez et al., 2014).

El objetivo fundamental de este documento será analizar cuáles han sido las dinámicas producidas en los usos de la comarca del Bierzo entre 1990 y 2012. Los objetivos específicos que se pretenden abordar, tienen relación con la observación de los procesos más importantes a los que se ha visto sometido este territorio por la tensión/dualidad existente, tanto en cubiertas arboladas debido a procesos de "forestación/deforestación", como aquellos que han provocado el aumento o descenso de la capacidad productiva en los usos agrícolas, principalmente afectados por procesos de "intensificación/extensificación". Por otro lado, debemos mencionar que el período posterior a 1990 es importante porque coincide con la entrada de España en la Unión Europea, lo que provoca que se modifiquen buena parte de las cubiertas de suelo en todo la comarca.

\section{Área de estudio}

La región del Bierzo tiene una extensión aproximada de 3 $183 \mathrm{~km}^{2}$ y está localizada al noroeste de la Comunidad Autónoma de Castilla y León. El área de estudio cubre la comarca administrativa de 38 ayuntamientos, según la delimitación establecida por la Ley 17/2010, de 20 de diciembre por la que se crea y se regula la Comarca de El Bierzo (Figura 1), bañada por la parte media y alta de la cuenca del Sil, cuya configuración se divide en una orla montañosa que culmina en 2,136 m y su altitud mínima se sitúa sobre los $350 \mathrm{~m}$. En torno a un $40 \%$ del territorio berciano se encuentra a una altitud inferior a $800 \mathrm{~m}$, lo que le permite poseer unas características bioclimáticas propias de ambientes mediterráneos (San Román, 2011).La actividad agraria del Bierzo posee una dualidad bien marcada entre las zonas de montaña y los fondos de valle. La ganadería tiene mayor peso que la agricultura en las primeras, donde los terrazgos se sitúan en lombas de pendiente menos pronunciadas, mientras que los fondos de valle son dedicados a cultivos de secano, huertas para autoconsumo y prados de siega principalmente. En las áreas de montaña, el abandono de tierras y los procesos de extensificación productiva son más evidentes. Por su parte, la Depresión del Bierzo tiene unas propiedades edáficas que le otorgan cierta ventaja para determinados cultivos, por ejemplo los pimientos, tomates y árboles frutales. Por este motivo, es en esta zona donde el paisaje agrario ha dado un cambio más acusado frente a los usos tradicionales en los últimos decenios, transformándose antiguas tierras de secano en favor de nuevos cultivos intensificados de regadío orientados hacia los mercados agro-industriales (San Román, 2011; Serrano et al., 2002).

En 1963, es cuando comienza la construcción de la red de infraestructuras hidráulicas del Bierzo, aparecen de este modo dos zonas de regadío bien definidas: el denominado Canal Bajo (5 800ha) y el Canal Alto (7 000ha). El primero de ellos, de topografía más plana donde los cultivos leñosos poseen una mayor fertilidad y grado de modernización. Más de un $30 \%$ de la superficie cultivada se encuentra en Toral de los Vados, Carracedelo, Cacabelos, Cabañas Raras y Camponaraya. Por el contrario, en el Canal Alto predomina el secano (prados- pastizales) (FernándezSoto et al., 2010).

Si observamos las áreas forestales de monte en el Alto Bierzo, las especies predominantes de las zonas de menor altitud son los robles (Quercus robur), encinas (Quercus ilex) y alcornoques (Quercus suber), mientras que en la montaña predominan los castaños rebollares (Quercus pyrenaica) y abedules (Populus alba). Por otro lado, en zonas llanas del Bierzo Bajo aparecen los usos de maderas nobles como el cerezo (Prunus avium) y nogal (Juglans regia) de producción maderera (Fernández \& Ramírez, 2004). Pero sin duda, la especie más abundante son las plantaciones de choperas (Populus spp.) que modifican enormemente el paisaje de toda la comarca. Otras masas como las pináceas poseen una gran productividad en zonas altas fuera de la Hoya central, sin embargo, las zonas bajas no son propicias para su desarrollo (Seara \& Fernández, 2011). También son frecuentes las especies de pino silvestre (Pinus sylvestris L.), laricio (Pinus nigra Arnold) y radiata (Pinus radiata $\mathrm{D}$. Don), este último analizado en la comarca por Rodríguez et al. (2005). 


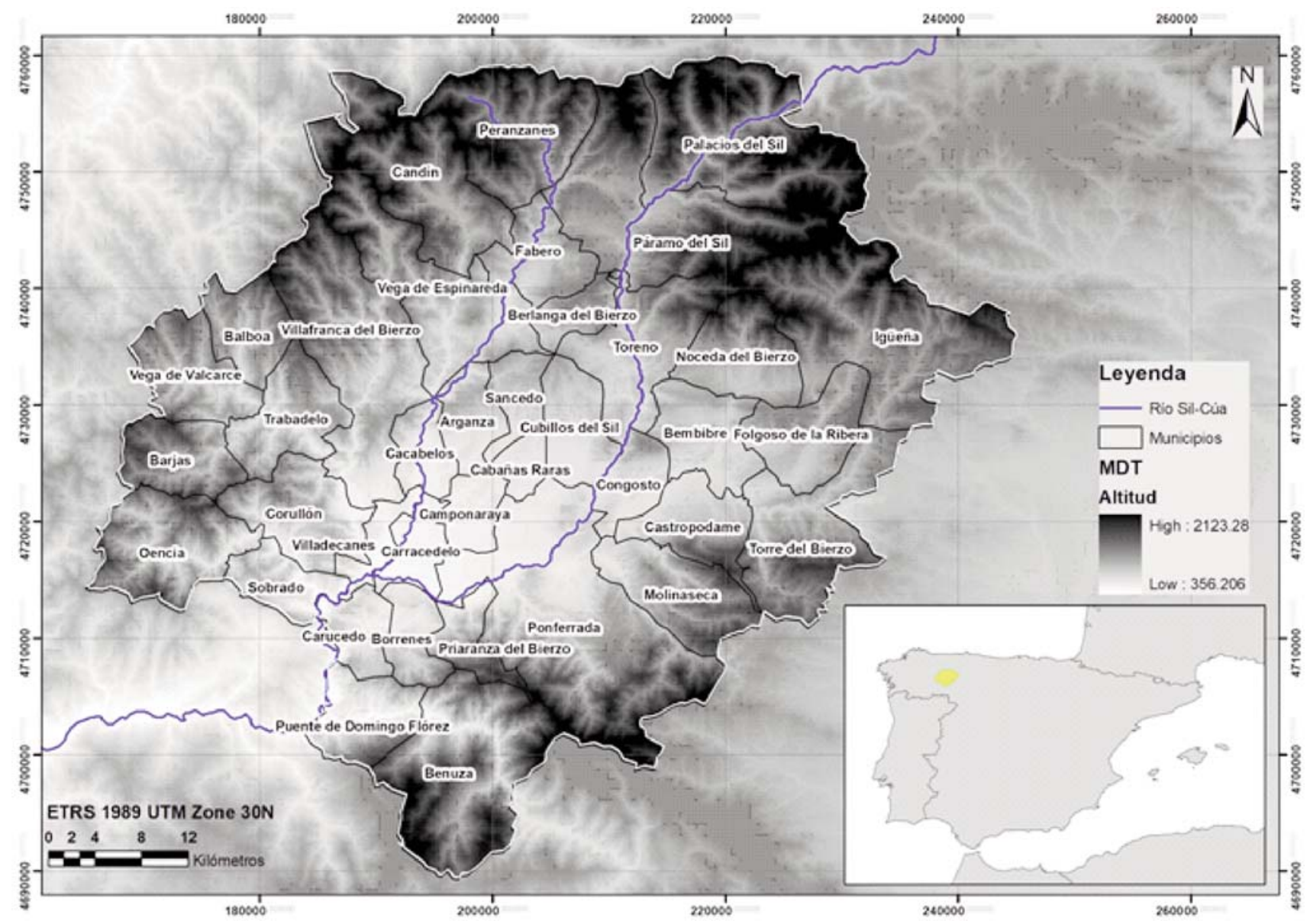

Figura 1.- Mapa de municipios de la Comarca del Bierzo. Elaboración propia

\section{Materiales y métodos}

Los materiales empleados en este trabajo parten del proyecto europeo Corine Land Cover para tres períodos diferentes (1990, 2000 y 2012) que han sido descargados del Instituto Geográfico Nacional y Copernicus. Para las tareas de análisis, hemos utilizado las aplicaciones libres GRASS GIS 7 (GRASS Development Team, 2016) y R (R Core Team, 2016).

La metodología empleada a través de CLC se corresponde por defecto con una escala 1:100 000 cuya unidad mínima cartografiable para los cambios de ocupación de suelo son 5 hectáreas. La comparación entre las diferentes ediciones se realizó en formato ráster con una resolución espacial de 25 metros de píxel. Para facilitar la interpretación de los datos, se ha realizado una reclasificación de la leyenda original del nivel tres (32 tipologías en el Bierzo) para formar 11 nuevas que hemos considerado dentro de este análisis visibles en la Tabla 1. A la hora de realizar la lectura sobre la metodología de Corine, se ha decidido incluir dentro de la categoría de las áreas de matorral la categoría de "improductivo", porque estas se corresponden principalmente con superficies quemadas. Otras categorías como la (243) áreas de espacios abiertos con bastante vegetación y (244) sistemas agroforestales se han categorizado como "uso agropecuario en extensivo", debido a las características del territorio en que nos encontramos.

\section{Resultados}

El balance general de los usos en 2012, muestra que la situación de las coberturas que ocupan mayor extensión son las masas de matorral y las cubiertas arboladas, cuya superficie se encuentra en torno al $47,5 \%$ y el $39,6 \%$ respectivamente sobre el total territorial. Por su parte, las cubiertas agrícolas se extienden solamente sobre el 9,7\%, y por último, las coberturas que menor superficie ocupan sobre el conjunto son las cubiertas artificiales $2,7 \%$ y las masas de agua $0,38 \%$ (Tabla 2). Si nos fijamos en el cómputo de los cambios entre las categorías analizadas y los resultados de Corine para nuestro intervalo de análisis, se observa la reducción de la superficie agrícola y arbolada, pero un aumento notable de la superficie de matorral. Durante el primer período (1990-2000) los valores muestran una menor variación porcentual y los cambios no son tan nítidos, mientras que, en el segundo intervalo (2000-2012) los usos se ven transformados con mayor intensidad (Tabla 2). 


\begin{tabular}{ccc}
\hline Código CLC (nivel 3) & L1 & L2 \\
\hline 212,213 & Regadio & \\
211 & Secano & Zonas agrícolas \\
231 & Prados-praderas & \\
$221,222,223,241,242$ & Cultivos permanentes & \\
243,244 & Agropecuario en extensivo & Matorral \\
\hline $321,322,323,324,332,333,334$ & Matorral & Arbolado \\
311 & Frondosas & \\
\hline 313 & Coniferas & Mas as de agua \\
\hline $411,511,512$ & Masas mixtas & Usos artificiales \\
\hline
\end{tabular}

Tabla 1.- Reclasificación de la leyenda original CLC. Fuente: Elaboración propia

\begin{tabular}{ccccccc}
\hline Uso/Cubierta & \multicolumn{2}{c}{ CLC 90 } & \multicolumn{2}{c}{ CLC 00 } & \multicolumn{2}{c}{ CLC 12 } \\
& Ha & $\%$ & Ha & $\%$ & Ha & $\%$ \\
\hline Regadio & 773 & 0,24 & 677 & 0,21 & 3530 & 1,11 \\
Secano & 1587 & 0,50 & 1413 & 0,44 & 775 & 0,24 \\
Prados-praderas & 4822 & 1,51 & 4293 & 1,35 & 7570 & 2,38 \\
Cultivos permanentes & 25356 & 7,96 & 25742 & 8,09 & 13143 & 4,13 \\
Agropecuario en extensivo & 14966 & 4,70 & 14713 & 4,62 & 5974 & 1,88 \\
\hline Áreas agrícolas & 47504 & 14,91 & 46838 & 14,71 & 30992 & 9,74 \\
\hline Frondosas & 72833 & 22,80 & 78956 & 24,80 & 98194 & 30,84 \\
Coniferas & 23885 & 7,50 & 26472 & 8,32 & 16231 & 5,10 \\
Masas mixtas & 38912 & 12,22 & 42929 & 13,48 & 11739 & 3,69 \\
\hline Area forestal & 135629 & 42,52 & 148357 & 46,60 & 126164 & 39,63 \\
\hline Matorral & 128519 & 40,37 & 113438 & 35,63 & 151459 & 47,57 \\
Superficies de agua & 1605 & 0,50 & 1605 & 0,50 & 1204 & 0,38 \\
Superficies artificiales & 5110 & 1,61 & 8129 & 2,55 & 8549 & 2,69 \\
\hline
\end{tabular}

Tabla 2.- Resultado de CLC para los períodos 1990, 2000 y 2012. Fuente: Elaboración propia

Los cambios netos en la evolución de las cubiertas forestales en este estudio muestran que el conjunto de las masas arboladas (coníferas, frondosas y masas mixtas) sufren un descenso del $2,97 \%$ sobre el total comarcal desde 1990 a 2012 (Tabla 2). Si nos centramos en los dos intervalos de estudio, durante el primero (1990-2000) la tendencia de las masas forestales era de aumento, en torno al $4 \%$, pero a inicios del nuevo siglo se revierte la situación al disminuir su superficie un 6,97\% (Tabla 2). A pesar de que el conjunto de las masas pasa a ocupar una menor superficie en 2012, este descenso se concentró sobre todo en la superficie de masas mixtas, que descienden 27189 ha y las masas puras de conífera 7653 ha, por otro lado se oculta la expansión de 25342 nuevas hectáreas de frondosa. El crecimiento de las masas de frondosa se explica por la expansión del chopo, donde llega a ocupar más del $30 \%$ de la superficie total del Bierzo en 2012, frente al 22,9\% que ocupaba en 1990 (Tabla 2). Sin embargo, para ese mismo año otras clases como las masas mixtas y coníferas ocupaban sólo el 3,69\% y el 5,1\% del total del Bierzo, respectivamente.

Por otra parte, sobre las áreas de matorral podemos apreciar que durante el estudio se produce un crecimiento en torno a un 7,21\%, alrededor de 23.000ha entre 1990 y 2012. Esta cobertura llega a ocupar la mitad de la superficie comarcal en 2012. Para los diez primeros años el matorral decrece, no obstante, para los doce restantes se produce el efecto contrario, creciendo 38 000ha (Tabla 2). Todo esto ha estado principalmente motivado por la extensificación productiva ocurrida sobre las cubiertas agrícolas.

En lo que respecta a los usos agrícolas, se observa que la superficie total dedicada a los cultivos desciende en el global, un 5,19\% desde 1990 (Tabla 2). Las categorías que sufren una mayor contracción son las áreas dedicadas a cultivos permanentes, las cubiertas extensivas y las áreas de secano. Sin embargo, aparece una tendencia a la expansión de la superficie de regadío y prados-praderas (Tabla 2). Como veremos posteriormente en las tablas de contingencia, la expansión o intensificación de la actividad agraria se produce más por una reorganización interna de los propios usos agrícolas, que por alternancias entre distintas categorías de la clasificación.

A la hora de analizar los procesos de expansión de las cubiertas artificiales en la comarca, las cifras muestran un ligero aumento para dicho período. Este crecimiento se produce principalmente durante los diez primeros años del estudio, donde aumenta un $0,95 \%$, mientras que en el segundo intervalo solo aumentan un $0,13 \%$ (Tabla 2). Para el año 2012 los usos artificiales cubren en torno a 8.500 hectáreas, motivadas principalmente por el incremento del tejido descontinuo dentro del área periurbana de Ponferrada y los municipios limítrofes a éste. Por último, situaríamos la categoría que menor porcentaje de superficie ocupa en el 
territorio berciano, como son masas de agua que abarcan sólo el $0,38 \%$, en ella se produce un ligero descenso desde la década de los noventa (Tabla 2).

Otro modo de extraer datos de nuestro análisis es recurrir a las tablas de contingencia (Tabla 3). En ellas aparecen las hectáreas transformadas y la evolución entre las distintas tipologías, donde aparece con mayor detalle los procesos ocurridos en cada caso, como veremos en los siguientes párrafos. En primer lugar, debemos fijarnos que para el intervalo (1990-2000) todas las categorías se mantienen estables en más de un $80 \%$, e incluso, seis de ellas superan el $90 \%$ lo que indica la estabilidad de las coberturas en la región para ese primer período (Tabla 3). Los verdaderos cambios se producen durante el segundo intervalo (20002012) donde el territorio berciano sí sufre mayores modificaciones. Podemos destacar que solo tres categorías (frondosas, masas de agua y matorral) se mantienen por encima del $65 \%$, las superficies artificiales también se sitúan por encima del $50 \%$ de superficie estable. Al contrario sucede con las restantes, porque las cubiertas agrícolas son las que soportan mayores transformaciones, puesto que ninguna supera el $20 \%$ de estabilidad, excepto los cultivos permanentes $35 \%$. Del mismo modo, las masas arboladas están sujetas a algún tipo de cambio de uso, hasta un $70 \%$ de las masas de coníferas y más de un $90 \%$ de las masas mixtas se transforman para dicho intervalo (Tabla 3). En los siguientes párrafos nos centraremos en explicar en profundidad los principales procesos que ocurren durante los 22 años del estudio.

El comportamiento de los usos forestales en las tres tipologías simples (coníferas, frondosas y mixtas) muestran tendencias divergentes entre ellas. Las que poseen una mayor estabilidad durante el transcurso del estudio son las frondosas $(66,9 \%)$. Por su parte, las coníferas presentan mayor variabilidad al mantenerse el $(29,1 \%)$. Por último, las masas mixtas son las que presentan mayores índices de cambio al transformarse más del $90 \%$ del total de la categoría (Tabla 3). Dentro de ellas, el proceso que más hectáreas modifica se produce por la permuta hacia masas de matorral desde las tres clases arboladas, en torno a 54 769 hectáreas se transforman desde 1990. En segundo plano, aparecen los cambios de composición entre las mismas, produciéndose el intercambio de alrededor de un $20 \%$ de la categoría de coníferas y mixtas hacia nuevas masas de frondosa, alrededor de 12000 hectáreas. De menor entidad, son las transformaciones hacia masas mixtas, donde cambian en torno a 3798 hectáreas procedentes tanto de arbolado de frondosas como de coníferas. Tan solo aparecen 1800 hectáreas de nuevas superficies dedicadas a coníferas procedentes de otras masas arboladas (Tabla 3).

En un segundo orden dentro del dominio forestal, las masas desarboladas tienen gran importancia sobre el marcado abandono que soporta esta región. Alrededor de 82247 ha del total de la categoría se mantienen desde 1990. Sobre el matorral aparecen más los procesos de reforestación que aquellos de regeneración natural sobre las propias masas, transformando para todo el período 39071 ha hacia nuevas cubiertas arboladas. Las frondosas son las que mayor superficie recibe por la plantación de choperas (21\%), seguido de las plantaciones de coníferas $(5,39 \%)$ y masas mixtas $(3,3 \%)$ respectivamente. Si analizamos el proceso contrario, el paso hacia nuevas superficies agrícolas se produce por la roturación de tierras, donde se transforman solo 2 913ha siendo los prados y cultivos permanentes los de mayor crecimiento (Tabla 3).

A la hora de tratar los procesos dentro de los usos agrícolas (cultivos permanentes, prados, regadío, extensivo y secano), después de ver la reducción de la extensión superficial de estos usos entre 1990-2012, también se percibe una gran inestabilidad dentro de las mismas. Un claro ejemplo se percibe con la categoría simple de secano que cambia completamente desde 1990.

En primer lugar, debemos analizar por separado las cubiertas agropecuarias en extensivo, ya que internamente sufren mayores índices de cambio debido a la transformación de casi la totalidad de la clase en 22 años, lo que provocó un descenso en superficie en todo el Bierzo. En esta clase, los principales procesos observados (por número de hectáreas) son: la extensificación por el paso hacia matorral de 5 255ha, los procesos de transición forestal permutando hacia masas arboladas 4 604ha. Por el contrario, se observan en menor medida procesos de expansión por roturación de nuevas áreas de cultivo, alrededor de $2545 \mathrm{ha}$, donde se transforman principalmente hacia cultivos permanentes (viñedo/frutales) y prados. También, aparecen procesos de intensificación productiva sobre 1250 ha de nuevas cubiertas irrigadas (Tabla 3 ).

Por otro lado, el proceso más notable en hectáreas que afecta a las restantes coberturas agrícolas sigue siendo el de extensificación productiva, esto se representa por el paso hacia matorral de (7 771 ha) y cubiertas agropecuarias extensivas (2 315 ha) procedentes de secano, cultivos permanentes y prados esencialmente. El segundo proceso percibido es el aumento de plantaciones de frondosa (chopo), aparecen gracias a los procesos de reforestación (4 486 ha), y siendo antiguas coberturas de prado y regadío las más afectadas por dicho proceso. Sin embargo, el proceso de intensificación productiva en la región es menos importante en cuanto a la superficie total transformada, no obstante, la clase de regadío aumentó 2853 ha desde el año 2000. Esta intensificación ocurre principalmente sobre terrenos procedentes de las categorías de prados $(9,7 \%)$, terrenos adehesados $(7,5 \%)$ y cultivos permanentes $(4,9 \%)$ (Tabla 3).

En lo relativo a las cubiertas artificiales, la expansión se produce como hemos visto en párrafos anteriores debido al incremento del número de polígonos industriales, la apertura de un elevado número de explotaciones mineras a cielo abierto, nuevas redes de infraestructuras, pero sobre todo el aumento del tejido urbano descontinuo. Se trata de una categoría bastante estable, ya que la mitad de la categoría se mantiene durante los 22 años del estudio. Aun así, observamos que la expansión se produce sobre zonas dedicadas anteriormente a matorral-monte bajo y zonas con vegetación abundante (3 232ha), sobre masas arboladas (2 008ha) y en menor medida destruyendo usos agrícolas (cultivos permanentes, prados y regadío) (1 280 ha) desde principios de los noventa (Tabla 3). 


\begin{tabular}{|c|c|c|c|c|c|c|c|c|c|c|c|c|}
\hline $1990-2000$ & Coniferas & C. Permanentes & A. Exiensivo & Frondasas & M.Agua & Masas mixias & Matomal & Prados & Regadio & Secano & Super. artificiales & Total ha (199) \\
\hline Coniferas & 22235 & 5 & 8 & 58 & 1 & 9 & 1309 & 2 & 1 & 0 & 259 & 23885 \\
\hline C. Permanentes & 18 & 24698 & 21 & 42 & 1 & 4 & 124 & 8 & 15 & 14 & 414 & 25358 \\
\hline A. Extensivo & 5 & 318 & 13990 & 171 & 1 & 28 & 289 & 1 & 29 & 1 & 135 & 14968 \\
\hline Frondosas & 23 & 127 & 19 & 69005 & 6 & 73 & 2374 & 10 & 3 & 40 & 554 & 72833 \\
\hline M.Agua & 0 & 1 & 1 & 4 & 1593 & 0 & 6 & 0 & 0 & 0 & 0 & 1606 \\
\hline Masas mixtas & 15 & 49 & 9 & 315 & 0 & 37773 & 724 & 3 & 0 & 0 & 24 & 38912 \\
\hline Matorral & 4152 & 160 & 392 & 8589 & 3 & 5014 & 107787 & 35 & 1 & 0 & 2387 & 128519 \\
\hline Prados & 1 & 332 & 1 & 113 & 0 & 5 & 59 & 4236 & 0 & 0 & 76 & 4822 \\
\hline Regadio & 0 & 24 & 63 & 6 & 0 & 0 & 29 & 0 & 629 & 1 & 21 & 773 \\
\hline Secano & 0 & 3 & 170 & 2 & 0 & 0 & 54 & 0 & 0 & 1358 & 0 & 1587 \\
\hline $\begin{array}{c}\text { Super ardficiales } \\
\text { Total ha }(2000)\end{array}$ & $\begin{array}{c}23 \\
28472\end{array}$ & $\begin{array}{c}27 \\
25742\end{array}$ & $\begin{array}{c}40 \\
14713\end{array}$ & $\begin{array}{c}53 \\
78950\end{array}$ & $\stackrel{0}{0}$ & $\frac{22}{42929}$ & $\begin{array}{c}685 \\
113438\end{array}$ & $\begin{array}{c}0 \\
4293\end{array}$ & $\begin{array}{c}0 \\
677\end{array}$ & $\begin{array}{c}0 \\
1413\end{array}$ & $\begin{array}{l}4260 \\
8129\end{array}$ & $\begin{array}{c}5110 \\
318387\end{array}$ \\
\hline $2000-2012$ & Coniferas & C. Pemmanentes & A. Extensivo & Frondosas & MAgua & Thasas mixtas & Mratomal & Prados & Regadio & Secano & Super. artibcales & Total ha $200 \mathrm{t}$ \\
\hline Coniferas & 8238 & 99 & 59 & 4972 & 2 & 2860 & 10187 & 9 & 0 & 0 & 88 & 28472 \\
\hline C. Permanentes & 193 & 9138 & 1997 & 2684 & 3 & 688 & 5631 & 3077 & 1254 & 280 & 939 & 25742 \\
\hline A. Extensivo & 204 & 1331 & 955 & 4023 & 6 & 245 & 5398 & 857 & 1115 & 138 & 441 & 14713 \\
\hline Frondosas & 686 & 781 & 908 & 52638 & 10 & 1273 & 20717 & $\$ 83$ & 329 & 80 & 604 & 78956 \\
\hline M. Agua & 7 & 4 & 4 & 250 & 1170 & 5 & 147 & 17 & 1 & 0 & 0 & 1606 \\
\hline $\begin{array}{c}\text { Masas mixtas } \\
\text { Matorral }\end{array}$ & $\begin{array}{l}1446 \\
5316\end{array}$ & $\begin{array}{c}127 \\
1038\end{array}$ & $\begin{array}{c}323 \\
1352\end{array}$ & $\begin{array}{l}9235 \\
21845\end{array}$ & $\begin{array}{l}0 \\
13\end{array}$ & $\begin{array}{l}2882 \\
3712\end{array}$ & $\begin{array}{l}28805 \\
77404\end{array}$ & $\begin{array}{l}149 \\
508\end{array}$ & $\begin{array}{c}0 \\
48\end{array}$ & 12 & $\begin{array}{c}189 \\
1425\end{array}$ & $\begin{array}{c}42929 \\
112428\end{array}$ \\
\hline Prados & 39 & 268 & 153 & 1518 & 0 & 22 & 1027 & $e 92$ & 467 & $\begin{array}{c}249 \\
0\end{array}$ & 109 & 4298 \\
\hline Regadio & 0 & 50 & 97 & 100 & 0 & 5 & 117 & 138 & 127 & 0 & 44 & 677 \\
\hline Secano & 0 & 107 & $\infty$ & 91 & 0 & 24 & 799 & 233 & 90 & 0 & 4 & 1413 \\
\hline Super. anficiales & 102 & 204 & 60 & 860 & 0 & 65 & 1458 & 530 & 100 & 15 & 4736 & 8129 \\
\hline Total ha $(2012)$ & 16231 & 13143 & 5974 & 98194 & 1204 & 11739 & 151459 & 7570 & 3530 & 775 & 8549 & 318387 \\
\hline $1990-2012$ & onifeas & Emanthes & A. Extensivo & Frandasas & MAgua & Masas mixtas & Matomal & Prados & Regadio & Secano & Super. artifiales & Total ha (199) \\
\hline Coníferas & 8964 & 82 & 50 & 4632 & 3 & 2860 & 9135 & 28 & 0 & 0 & 330 & 23885 \\
\hline C. Permanentes & 169 & 9120 & 1966 & 2474 & 3 & 681 & 5488 & 2872 & 1249 & 281 & 1085 & 25356 \\
\hline A. Extensivo & 283 & 1245 & 886 & 4090 & 5 & 321 & 5255 & 1019 & 1128 & 137 & 598 & 14968 \\
\hline Frondosas & 618 & 704 & 902 & 48728 & 9 & 1138 & 18544 & 868 & 354 & 81 & 890 & 72833 \\
\hline ML Agua & 7 & 4 & 4 & 249 & 1171 & 5 & 148 & 17 & 1 & 0 & 0 & 1606 \\
\hline Masas mixtas & 1184 & 79 & 251 & 7363 & 0 & 2804 & 27090 & 137 & 0 & 13 & 191 & 38912 \\
\hline Matonal & 6923 & 1239 & 1545 & 27990 & 13 & 4245 & 82247 & 1351 & 76 & 247 & 2634 & 128519 \\
\hline Prados & 39 & 305 & 190 & 1759 & 0 & 22 & 1146 & 715 & 471 & 0 & 177 & 4822 \\
\hline Regadio & 0 & 105 & 103 & 155 & 0 & 5 & 128 & 134 & 124 & 0 & 18 & 773 \\
\hline Secano & 1 & 96 & 68 & 98 & 0 & 24 & 1009 & 232 & 60 & 1 & 0 & 1587 \\
\hline $\begin{array}{c}\text { Super, ardificiales } \\
\text { Total ha }(2012)\end{array}$ & $\begin{array}{c}43 \\
16231\end{array}$ & $\begin{array}{c}163 \\
13143\end{array}$ & $\begin{array}{l}20 \\
5974\end{array}$ & $\begin{array}{c}847 \\
98194\end{array}$ & $\stackrel{0}{1204}$ & $\begin{array}{c}55 \\
11739\end{array}$ & $\begin{array}{c}1289 \\
151459\end{array}$ & $\begin{array}{c}200 \\
7570\end{array}$ & $\begin{array}{c}69 \\
3530\end{array}$ & $\frac{17}{775}$ & $\begin{array}{l}2827 \\
8549\end{array}$ & $\begin{array}{c}5110 \\
318387\end{array}$ \\
\hline
\end{tabular}

Tabla 3.- Tablas de contingencia para los tres períodos en hectáreas. Fuente: Elaboración propia

\section{Discusión}

Los cambios descritos tuvieron lugar es un período de relativa estabilidad de la población total, aunque se produjo un ligero descenso de 2072 efectivos de población, se sitúan por encima de los 130000 habitantes en el año 2012 (INE, 2016). Pero los verdaderos cambios que nos preocupan en nuestro período analizado, han sido los movimientos migratorios interiores, principalmente hacia la capital y núcleos cabecera, que son los que realmente han condicionado el aumento de efectivos en los municipios limítrofes a Ponferrada, que funcionan como foco de atracción, dejando así despobladas las zonas rurales periféricas a esta (Monteserín, 2006; Lois et al., 2008).

Después de haber visto los resultados de este análisis, los procesos que más han afectado a los cambios de uso/cobertura son: la extensificación productiva y el abandono de la actividad humana para el período 19902012. Las áreas más alejadas de los núcleos de población y aquellos municipios con zonas de fuerte pendiente son los que sufren mayor abandono, al igual que sucede en Galicia (Corbelle \& Crecente, 2008), aunque en zonas de vega cercanas al río Sil también se percibe dicho proceso (Fernández-Soto et al., 2010). Por otra parte, son destacables aunque con poca extensión superficial, los procesos de intensificación muy localizadas en áreas cercanas a las infraestructuras hidráulicas del Canal del Bierzo (Fernández-Soto et al., 2010; Cortizo, 2001). Este aumento de las áreas regadas se debe al cambio en diversos cultivos (es posible que aumenten en los próximos años debido al aumento de la fruticultura en regadío) y al crecimiento de la superficie dedicada a prados, apareciendo como respuesta a la desaparición de las cubiertas de secano en toda la comarca (San Román, 2011).

Los datos que realmente sorprenden se asocian al crecimiento superficial de las frondosas dentro del estudio, parte de este incremento se produce por la plantación de chopos en zonas de vega, donde se ocupan los terrenos más aptos para el cultivo dentro de la Hoya berciana y áreas próximas al río Sil-Cúa. Todo este proceso de reforestación se ha producido sin organización lógica y con escaso control por parte de la administración local (Fernández \& Ramírez, 2004). La aparición de las medidas europeas bajo el Reg. 2080/92 de forestación de tierras agrarias han podido condicionar este proceso. No obstante, no descartamos que también se produzca un aumento de quercíneas en zonas de la orla montañosa, lo que provoca la transformación de buena parte del paisaje forestal sobre todo a partir del año 2000 por regeneración espontánea de la vegetación. 
En relación con lo mencionado anteriormente, se percibe que existe una falta de gestión forestal, principalmente en el Alto Bierzo y zonas montañosas de la orla exterior, junto con los procesos de transición forestal que afectan sobre todo a las frondosas, en menor medida a otras masas. Esto ha provocado un cierto grado de abandono en las cubiertas forestales, tanto arboladas como desarboladas, lo que ha generado una serie de problemáticas asociadas a los incendios forestales que se produjeron sobre todo durante el segundo intervalo (2000-2012), llegando a provocar graves problemas en todo el territorio (Fernández \& Ramírez, 2004). La posible corta de algunas plantaciones forestales ha favorecido su posterior abandono, y de este modo propician también el crecimiento por regeneración espontánea de nuevas hectáreas de matorral acrecentando dicha problemática y riesgo. Según el trabajo de Serrano et al. (2002) las masas forestales eran la categoría de uso que más porcentaje ocupaba dentro de la comarca (aproximadamente $42 \%$ del total en 1999). Actualmente, los usos forestales tienen buenas aptitudes para la producción, pero en las zonas repobladas las técnicas silvícolas no son las más adecuadas, lo que implica un importante cambio estructural para conseguir un correcto aprovechamiento (Rodríguez et al., 2005).

Observando los datos de la superficie artificializada en la región, la ocupación de estos usos en la Comunidad de Castilla y León para el año 2000 , se situaban en torno al $1,3 \%$ (OSE, 2006). En nuestro caso, el Bierzo para ese mismo período las cifras rondan el $2,55 \%$ de ocupación, este crecimiento de las cubiertas artificiales ha sido evidente, pero no tan elevado como se esperaba en un principio. Debemos tener en cuenta que el Bierzo es una zona esencialmente rural, con una tipología de poblamiento disperso esparcido por toda la región (Monteserrín, 2006). Debido a este factor el aumento de estas cubiertas no ha sido tan brusco como en otras áreas españolas, aunque la concentración de efectivos de población ha provocado que algunos municipios como Toreno, Camponaraya, Cacabelos, Fabero, Cubillos del Sil, Bembibre y Ponferrada crezcan.

Si nos fijamos en los datos del trabajo de Serrano et al. (2002) procedentes del Servicio Territorial de Estadística de la Junta de Castilla y León, para el período 1990-1999, muestran cifras similares a nuestro primer intervalo (19902000) sobre la distribución de usos en la región. Ellos demuestran, por ejemplo, que la superficie cultivada decrece un $2,6 \%$ sobre el total pero aumentan ligeramente la extensión de prados y pastizales un 1\%. En 1999, la distribución de tierras labradas está ocupada principalmente por viñedo (42,95\%), seguidas de los frutales $(34,24 \%)$ y cultivos herbáceos $(22,78 \%)$ (Cortizo, 2001). Son otros artículos los que analizan cambios de usos en regiones próximas, como en Galicia (Corbelle \& Crecente, 2014; Díaz et al., 2005) donde se perciben tendencias similares en cuanto al abandono y extensificación de usos agrícolas. También son visibles en el norte de Portugal (Aguilera \& Botelquilha, 2012), incluso autores que han utilizado Corine perciben el incremento de las masas de frondosa en zonas cercanas al río Duero (Martínez-Fernández et al., 2014). Para finalizar este apartado, la modernización de la superficie agrícola utilizada (SAU) del Bierzo es nítida, pero existen varios factores que merman la productividad y el crecimiento real de la comarca, como son: la inexistente ordenación territorial desde mediados del S. XIX (Burgueño, 1995), la elevada fragmentación de la propiedad, tanto de parcelas agrícolas como forestales (Cortizo, 2001; Fernández \& Ramírez, 2004; Rodríguez et al., 2006), como sucede en otras provincias del Noroeste peninsular que limitan con esta (Calvo et al., 2009). Otra problemática importante aparece debido al descenso de la cabaña ganadera y la reducción drástica del número de explotaciones en la zona, como se muestra en la comparativa de los Censos Agrarios de 1999-2009 (Figura 2). Para dicho período se produce un descenso del $63,1 \%$, alrededor de 4070 explotaciones, aunque debemos mencionar que el cambio de metodología entre ambos períodos (donde no se contabilizan las explotaciones que tengan menos de una hectárea a partir del año 1999). De este modo, se puede sobreestimar la definición del universo censal, lo que puede influir en la caída drástica del número de explotaciones de la comarca, como se ve reflejado en la (Figura 2). Tampoco podemos olvidar los factores orográficos como verdaderos limitantes sobre cultivos de elevada pendiente, que han afectado más a los municipios de orla montañosa en Gistredo, Sierra de Ancares y los Montes Aquilanos.

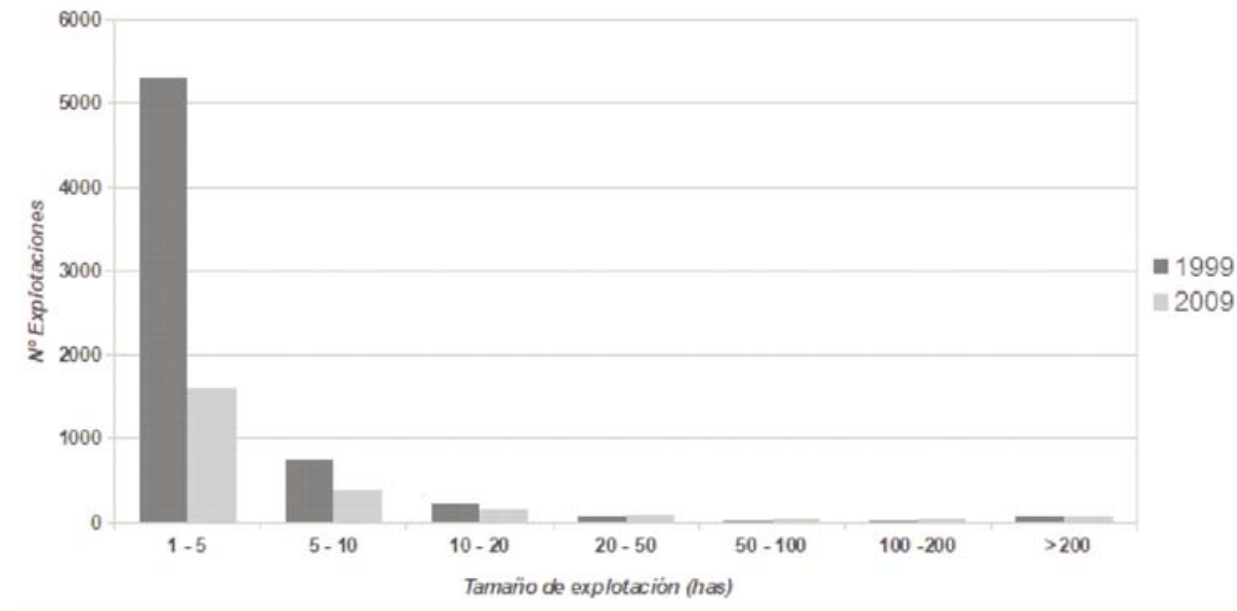

Figura 2.- Número de explotaciones y tamaño medio de explotación. Fuente: INE, Censo Agrario 1999-2009 
En último caso, debemos tener en cuenta los efectos derivados de los problemas de la escala de representación de Corine, ocasionando problemas a la hora de analizar categorías que ocupan poca superficie dentro del territorio berciano, lo que ha podido alterar una pequeña parte de nuestros datos. El tamaño de la unidad mínima cartografiable (5ha) es mayor que el de la mayoría de las parcelas de esta comarca. De este modo, una parte de los cambios operados en las parcelas puede pasar desapercibida. Como aspecto a destacar, no existe ninguna fuente actualmente que nos permita analizar un período temporal tan largo (22 años) o existen pocas alternativas. La única opción plausible para analizar esta comarca sería el Mapa de Cultivos y Aprovechamientos con una escala 1:50 000 .

\section{Conclusiones}

Podemos concluir que se producen una serie de cambios de uso/cobertura para el período temporal analizado que han provocado serias transformaciones en el paisaje agrario, urbano y forestal del Bierzo. Se ha podido apreciar que los procesos más acusados, sin duda son el grave abandono que sufre esta comarca motivado por las transformaciones del número de hectáreas totales hacia matorral, procedentes sobre todo de los usos forestales 54 769ha y agrícolas 13 026ha. Casi la mitad de la superficie del Bierzo pertenece al dominio del matorral en 2012, puesto que ocupa en torno a 151 000ha lo que plantea un serio problema para la gestión de los incendios forestales en la zona. En otro orden, el aumento de las plantaciones de chopo por reforestación también ha sido importante, muestra de ello se refleja por el aumento porcentual del $7,9 \%$ de las frondosas desde 1990. Los procesos de intensificación productiva aparecen cerca de las zonas irrigadas del Bierzo, aunque no son tan importantes sobre el total regional.

Para finalizar, como sociedades modernas debemos ejercer un mejor uso de nuestro territorio, intentando paliar los problemas que aparecen en ellos. Muestra de ello, se encuentra este territorio cada vez más improductivo y fragmentado, cuyos problemas son visibles en la actualidad y requieren de cambios en su Ordenación Territorial. Por este motivo, deben implicarse más los organismos públicos y los propietarios para tratar de mitigar algunos de estos aspectos en el futuro. Aunque en los últimos años, el Bierzo parece que quiere dar un giro hacia la industria agroalimentaria, gracias a diversas denominaciones de origen protegidas, que pueden impulsar la aparición de nuevas estructuras agrarias y la expansión de nuevos cultivos para que vuelvan a tener importancia en el Bierzo, o por lo menos, ayuden al mantenimiento económico de la comarca.

\section{Bibliografía}

Aguilera, F. \& Botelquilha, A. (2012). Selección de métricas de paisaje mediante análisis de componentes principales para la descripción de cambios de uso y cobertura del suelo del Algarve, Portugal. Geofocus 12: 93-121.

Alberdi, J. (2001). Reestructuración agraria y abandono de usos: El caserío vasco. Investigaciones geográficas 26 : $135-150$

Burgueño, J. (1995). La génesis de la división territorial contemporánea en la España atlántica (Galicia, Asturias, Cantabria y El Bierzo). Ería 36: 5-33.

Corbelle, E. \& Crecente, R. (2008). El abandono de tierras: Concepto teórico y consecuencias. Revista Galega de Economía Vol.17, 2.

Corbelle, E. \& Crecente, R. (2014): Urbanización, forestación y abandono. Cambios recientes en el paisaje de Galicia. Revista Galega de Economía Vol. 23, 1: 35-52.

Corbelle, E. J., Butsic, V., Enríquez, M. J. \& Volker, C. (2015). Technology or policy? Drivers of land cover change in northwestern Spain before and after the accession to European Economic Community. Land Use Policy 45: 1825.

Cortizo, J. (2001). El Bierzo. Hacia la modernización agraria. Repositorio Institucional Abierto (BULERIA).

de Aranzabal, I., Schmitz, M., Aguilera, P. \& Pineda, F. (2008). Modelling of landscape changes derived from the dynamics of socio-ecological systems: A case of study in a semiarid Mediterranean landscape. Ecological Indicators 8: $672-685$.

Díaz, J. M., Aller, D., Martín, A., Barcia, B. \& Pereira, S. (2005): Dos perspectivas sobre la cartografía de coberturas y usos de suelo en Galicia. Revista Galega de Economía Vol.16, 1.

Fernández, A. \& Ramírez, J. (Coords.) (2004): El alto Bierzo. Una propuesta forestal sostenible. Junta de Castilla y León.

Fernández-Soto, M., García, A., Cuesta, F. \& Prieto, J. R. (2010). El sistema de regadío del Bierzo. Ería 82: 159-169.

GRASS Development Team (2016). Geographic Resources Analysis Support System (GRASS). Software, Version 7.2.0. Open Source Geospatial Foundation. http://grass.osgeo.org

Jaraíz, F. J., Mora, J. \& Gutiérrez, J. A. (2012). Cambios de cobertura y uso del suelo en la Raya Central Ibérica. Boletín de la A.G.E. 60: 507-514.

Jones, N., De Graaf, J., Rodrigo, I. \& Duarte, F. (2011). Historical review of land use changes in Portugal (before and after EU integration in 1986) and their implications for land degradation and conservation, with a focus on Centro and Alentejo regions. Applied Geography 31: 1036-1048. 
Lambin, E. F., Turner, B.L., Geist, H., Agbola, S., Angelsen, A., Bruce, J. Coomes, O \& Folke, C. (2003). The causes of land-use and land-cover change: moving beyond the myths. Global Environmental Change 11,4: 261-269.

Lasanta, T. (2000). Cambios de uso en el regadío de la Rioja durante la segunda mitad del S.XX: de la intensificación a la extensificación. Aróspide, 355-368.

Levers, C., Butsic, V., Verburg, P., Müller, D. \& Kuemmerle, T. (2016). Drivers of Changes in Agricultural Intensity in Europe. Land use policy 58: 380-393.

Lois, R. C., San Román, J. M. \& Aldrey, J. A. (2008). Impacto de la actividad minera en la población de los espacios de montaña. La pizarra y en carbón en las montañas galaicoleonesas. Ería 75, 99-112.

Lomba, A., Vicente, J., Moreira, F. \& Honrado, J. (2011) Effects of Multiple Factors on Plant Diversity of Forest Fragments in Intensive Farmland of Northern Portugal. Forest Ecology and Management 262 (12): 2219-2228.

Macdonald, D., Crabtree, J.R., Fleury, P., Dax, T., Wiesinger, G., Stamou, N., Gutiérrez, J. \& Gibon, A. (2000). Agricultural abandonment in mountain areas of Europe: environmental consequences and policy. Journal of Environmental Management, Vol. 59, 1: 47-69.

Martínez, J., Sánchez, N. \& Celaya, L. (2014). Evolución de la cubierta forestal de la cuenca del Duero: análisis multitemporal mediante teledetección. XVI Congreso Nacional de Tecnologías de la Información Geográfica. (25,26 y 27 de Junio). Alicante.

Meyfroidt, P., Lambin, E. F., Erb, K. H. \& Hertel, T. W. (2013). Globalization of Land Use: Distant Drivers of Land Change and Geographic Displacement of Land Use. Curr. Opin. Environ. Sustain. 5: 438-444

Molinero, F., Tort, J., Ojeda, J., Ruíz, E., Martínez, E., Silva, R. \& Mata, R. (2013) (Coords.). Atlas de los paisajes agrarios de España. Ministerio de Agricultura, Alimentación y Medio Ambiente. Madrid.

Monteserín, O. (2006) Los procesos de transformación urbana y la producción de imagen de la ciudad. El caso de Ponferrada. Biblioteca Virtual Miguel de Cervantes (Alicante).

Observatorio Sostenibilidad de España (2006). Cambios de ocupación de suelo en España. Implicaciones para la sostenibilidad. Observatorio de la Sostenibilidad en España. Ministerio del Medio Ambiente. Mundi-Prensa, 485.
Plieninger, T., Draux, H., Fagerholm, N., Bieling, C., Bürgi, M., Kizos, T., Kuemmerle, T., Primdahl, J. \& Verburg, P. (2016). The Driving Forces of Landscape Change in Europe: A Systematic Review of the Evidence. Land use policy 57: 204-214.

San Román, J. M. (2011). La diversidad de los paisajes de fruticultura en el Bierzo: La pujanza de los regadíos de manzanos y perales y la conservación de "los sotos" de castaños. Polígonos Revista de Geografía 21: 119-148.

Seara, M. P. \& Fernández, A. (2011). Análisis y diagnóstico territorial de la populicultura en la comarca del BierzoLeón. Spanish journal of rural development, Vol.2: 33-54.

Serra, P., Vera, A., Francesc, A. \& Salvati, L. (2014): Beyond urban-rural dichotomy: exploring socioeconomic and landuse processes of change in Spain (1991-2011). Applied Geography Vol. 55: 71-81.

Serrano, E., Lavín, P. \& Mantecón, A. R. (2002). Censos de ganado y de explotaciones, distribución de la población y uso del territorio en la provincia de León: Situación actual y evolución. Polígonos. Revista de Geografía 11-12: 167197.

R Developmmento Core Team (2016). A language and environment for statistical computing. R Foundation for Statistical Computing, Vienna, Austria. http://www.Rproject.org

Reglamento (CE) N²080/92 del Consejo de 30 de junio de 1992, por el que se establece un régimen comunitario de ayudas a las medidas forestales en la agricultura. Diario oficial de la Unión Europea 30/7/92

Rodríguez, J. R., Álvarez, M. F., Asenjo, A., Fernández, A., \& Quintano, C. (2005). Inventariación de las masas de Pinus Radiata D.Don en el Bierzo (León) empleando la Teledetección con imágenes multiespectrales de resolución media. Cuadernos de la Sociedad Española de Ciencias Forestales 19: 175-180.

Turner, B.L., Lambin, E. \& Reenberg, A. (2007). The emergence of land change science for global environmental change and sustainability. Proceedings of the National Academy of Sciences (PNAS) Vol. 104, 52.

van Vliet, J., de Groot, H. L. Rietveld, P. y Verburg, P.H. (2015). Manifestations and underlying drivers of agricultural land use change in Europe. Landscape and Urban Planning 133: 24-36. 